Relations industrielles

Industrial Relations

\title{
Whitfield, Keith et George Strauss (sous la direction de), Researching the World of Work - Strategies and Methods in Studying Industrial Relations
}

\section{Sylvie Montreuil}

\section{Volume 54, numéro 1, 1999}

Relations industrielles et nouveaux systèmes productifs

Industrial Relations in the New Workplace

URI : https://id.erudit.org/iderudit/051227ar

DOI : https://doi.org/10.7202/051227ar

Aller au sommaire du numéro

Éditeur(s)

Département des relations industrielles de l'Université Laval

ISSN

0034-379X (imprimé)

1703-8138 (numérique)

Découvrir la revue

Citer ce compte rendu

Montreuil, S. (1999). Compte rendu de [Whitfield, Keith et George Strauss (sous la direction de), Researching the World of Work - Strategies and Methods in Studying Industrial Relations]. Relations industrielles / Industrial Relations, 54(1), 201-203. https://doi.org/10.7202/051227ar

Tous droits réservés (C) Département des relations industrielles de l'Université Laval, 1999
Ce document est protégé par la loi sur le droit d'auteur. L'utilisation des services d'Érudit (y compris la reproduction) est assujettie à sa politique d'utilisation que vous pouvez consulter en ligne.

https://apropos.erudit.org/fr/usagers/politique-dutilisation/ 
auteurs ont éloquemment illustré l'importance de la communication entre toutes les parties nécessairement tributaires de l'opération. L'information donnée d'une façon claire, honnête et continuelle permet d'éviter des réactions vives de certains, d'éliminer les rumeurs dévastatrices et, surtout, elle manifeste un réel respect des autres. Ces mêmes auteurs signalent également comment de véritables programmes de formation professionnelle peuvent être salutaires et pour les salariés et pour les entreprises. En somme, ce temps de formation à une période de transition peut faciliter l'attente du nouveau départ et permettre à l'entreprise de disposer d'une maind'œuvre qualifiée, fidèle et dévouée. Outre le respect de tous les véritables partenaires de l'entreprise et de l'importance de la prise d'une décision d'une manière responsable, les auteurs n'ont guère traité directement de l'éthique. Ce volet est abordé davantage par voie d'illustrations de ce qu'est et de ce que ne pourrait être une décision d'un gestionnaire respectueuse des intérêts de tous (actionnaires, gestionnaires, salariés, clients, fournisseurs et société), toutes choses bien considérées. En d'autres termes, on a préféré l'évidence tirée de l'expérience à la remontrance moralisatrice. Il nous serait difficile de contester ce choix.

FERNAND MORIN

Université Laval

\section{Researching the World of Work - Strategies and Methods in Studying Industrial Relations}

publié sous la direction de Keith WHITFIELD et George STRAUSS, New York: Cornell University Press, 1998, 330 p., ISBN 0-8014-8549-5.

Cet ouvrage vise à exposer et à questionner les stratégies de recherche et les méthodes utilisées en relations industrielles. Le public cible s'avère les chercheurs, les praticiens et les étudiants qui souhaitent mieux comprendre l'évolution de la recherche et des études en relations industrielles et les choix méthodologiques qui s'offrent à eux pour en approfondir la compréhension. Il s'agit d'un collectif où 22 auteurs, mandatés par le Research Methods Study Group of the International Industrial Relations Association, fournissent un tour d'horizon plutôt exhaustif et dynamique de ce qui s'est fait et de ce qui peut être fait dans l'étude empirique des relations industrielles. Au début du livre, les directeurs expliquent que la nécessité de procéder à la publication de cet ouvrage provient du constat suivant : jusqu'à récemment la plupart des recherches en relations industrielles privilégiaient, en grande partie, l'étude des relations patronales-syndicales mais, depuis quelques années, le spectre s'élargit autour des enjeux de performance du monde du travail laquelle inclut la santé et la sécurité du travail, les pratiques managériales, la discrimination, la satisfaction des employés, la sécurité d'emploi et l'analyse comparative des systèmes de relations industrielles. Ces thèmes ne sont pas nouveaux certes, mais leur étude a généré encore plus d'interactions avec des chercheurs d'autres disciplines. De plus, les auteurs constatent une plus grande variété de stratégies et de méthodes utilisées pour répondre aux questions de recherche du monde contemporain des relations industrielles.

Bien que ce livre puisse se voir attribuer l'étiquette de théorique, il ne discute pas de forces ou de faiblesses de modèles théoriques utilisés en relations industrielles, il ne tient pas non plus à faire de débat sur les relations industrielles en tant que champ ou discipline. Cependant, un puissant fil conducteur tisse des liens solides entre les chapitres: la recherche empirique, soit celle qui repose sur l'étude de faits et ce, qu'il s'agisse du champ d'étude ou d'une discipline particulière au sein des relations industrielles. 
D'emblée, ce livre pose les deux principales approches de la recherche en relations industrielles: les approches inductives et déductives. Les lecteurs apprécieront sans doute l'argumentation posant les avantages et les inconvénients de chacune d'elles, mais surtout la nécessité d'avoir recours à ces deux approches compte tenu de la diversité des thèmes à l'étude. Et il en va de même pour l'attrait des méthodes qualitatives et quantitatives. Les directeurs et les auteurs posent plusieurs réflexions prospectives quant au devenir des méthodes de recherche en relations industrielles compte tenu de l'évolution des thèmes des dernières années et des transformations du monde du travail.

Le cour de cet ouvrage tient en quatre grandes sections. La première expose les enjeux actuels des champs d'étude en relations industrielles et tente de préciser les principales stratégies et méthodes de recherche qui s'y appliquent: les méthodes qualitatives, quantitatives, expérimentales, et l'étude de cas. De plus, deux chapitres portent sur l'apport de l'ethnographie dans l'étude du monde du travail et de la recherche-action de type participatif. Généralement, il s'agit de décrire une méthode ou une stratégie avec, à l'appui, des illustrations de travaux de recherche qui les ont utilisées. Certains chapitres mettent en évidence les expériences " terrain * des chercheurs devant les difficultés rencontrées ou les limites de leur contribution. Le texte est, en général, fort explicite sur les avantages et les limites des stratégies et méthodes décrites. Plusieurs états de la question s'avèrent fort instructifs quant aux origines et à l'évolution de nombreuses pratiques en relations industrielles, comme les enquêtes nationales et celles portant sur les attitudes des employés.

Une deuxième section est consacrée à l'analyse comparative des systèmes à partir de données nationales ou d'études de cas. L'argumentaire sur la nécessité de conduire de telles études est convaincant et les stratégies de recherches retenues y demeurent clairement présentées. Cette section, à l'image du livre, a le mérite de montrer la complémentarité des sources des données et des approches dans l'étude de phénomènes complexes.

Une place privilégiêe est accordée à l'apport des méthodes utilisées dans trois disciplines qui contribuent à mieux comprendre les relations qui s'établissent entre les acteurs du monde du travail : l'histoire, le droit et la sociologie. Ici, un effort particulier a été investi pour situer les fondements disciplinaires de ces approches, leurs points d'ancrage par rapport aux relations industrielles et l'intérêt de leur contribution à la compréhension des régulations s'opérant dans le monde du travail. Finalement, bien que plusieurs chapitres abordent l'intérêt de la recherche empirique pour mieux élaborer les politiques publiques en matière de travail, une section porte spécifiquement sur ce sujet en mettant en perspectives certaines faiblesses associées à l'utilisation de données ou de modèles plus ou moins pertinents pour établir des politiques concernant le marché du travail à partir d'exemples étasuniens.

Somme toute, ce livre apparait comme une synthèse fort éclairante du rôle de la recherche empirique en relations industrielles à partir du passé et du présent. La plupart des textes mettent l'emphase sur les aspects prospectifs de sa contribution compte tenu de l'évolution actuelle du monde du travail. Un réel effort a été fait pour rendre le contenu représentatif de l'ensemble de la situation au niveau international, mais les pôles d'attraction demeurent les réalisations anglaises et américaines avec une place non négligeable accordée à l'Océanie.

Ce livre amc̀nc le lecteur à la conclusion que la recherche empirique en relations industrielles diversifie de plus en plus ses stratégies et ses méthodes tout en favorisant la contribution de chercheurs d'autres disciplines, et ce phénomène est 
notamment tributaire de la division théorique actuelle de l'étude du monde du travail. Le risque que la recherche en relations industrielles perde de sa spécificité existe, mais les auteurs y voient une occasion d'utiliser ces apports pour renforcer les théories et les bases conceptuelles propres au monde du travail et à ses régulations. Cet ouvrage s'avère le premier du genre et constitue un outil sérieux, fort bien documenté et particulièrement réussi à montrer la cohérence et les défis qui s'annoncent dans l'étude des faits dans le monde du travail, tel que cerné en relations industrielles.

SYLVIE MONTREUIL. Université Laval

New Forms of Work Organization - Can Europe Realise its Potential? by EPOC RESEARCH GROUP, Dublin: The European Foundation for the Improvement of Living and Working Conditions, 1997, ISBN 92-828-1888-8.

As in North America, there has been considerable interest within the European Union (EU) in the diffusion and effects of various workplace "innovations," with many convinced that these innovations benefit both employees and employers and that they offer the potential for a transformation to a new, "social partnership" model of work organization and employment relations. These innovations involve "direct" rather than more traditional, representational forms of participation, either through the reorganization of work so that extended rights and responsibilities are granted to employees (e.g., autonomous groups), or through increased consultation with employees (e.g., suggestion schemes, quality circles).

This book represents an interim report on a 1996 survey of employers in ten EU countries as to nature and extent of both delegative and consultative forms of direct participation, the motives underlying their implementation, their links with organizational strategies, their effects on employers and employees, how they are implemented and managed, and what their relationships are with employee qualifications, training, and remuneration systems. The survey involved a questionnaire consisting of (by my count) well over 200 items, and mailed to the general manager in 33,427 workplaces across the ten countries. It was sponsored by the European Foundation for the Improvement of Living and Working Conditions, an EU funded research institute located in Dublin, and was conducted by an eleven member research team, coordinated by Keith Sisson of Warwick.

In smaller countries (Ireland, Portugal, Denmark, the Netherlands, Sweden) workplaces with less than 20 employees were excluded, while in larger countries (France, Germany, Italy, Spain, the U.K.), the cut-off was 50 employees. The questions were targeted at the largest occupational group in each workplace, and the sampling was random, including both public and private sector workplaces. Though it varied considerably by country (from 9.8 percent in Spain, to 38.8 percent in Ireland), the overall response rate was 17.8 percent, yielding a final sample of 5,786 workplaces. The data were weighted on the basis of sector, workplace size, and national labour force size in order to eliminate sample distortions.

The main findings as to the extent and intensity of direct participation are largely consistent with those from similar surveys in Canada and the U.S. Though more than four out of five of those workplaces participating in the survey had some form of direct participation, only about one in seven had adopted more than a few forms, and the scope of participation was typically limited; the authors calculate that only about two percent appear to have undergone a 